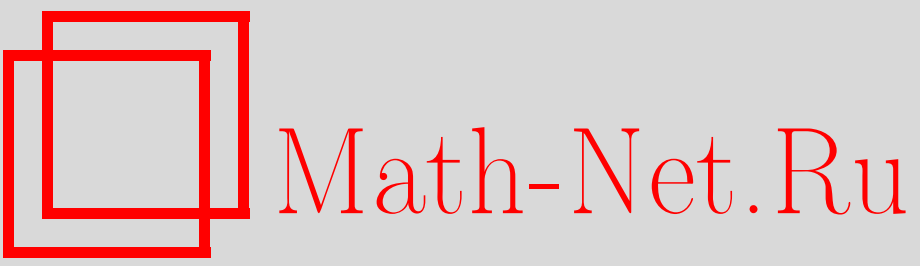

П. В. Гапеев, Байесовские задачи последовательного различения гипотез для непрерывных случайных процессов, УМН, 2000, том 55, выпуск 1, 177-178

DOI: https://doi.org/10.4213/rm255

Использование Общероссийского математического портала Math-Net.Ru подразумевает, что вы прочитали и согласны с пользовательским соглашением

http://www . mathnet.ru/rus/agreement

Параметры загрузки:

IP : 54.198 .187 .58

26 апреля 2023 г., 04:18:47 


\title{
БАЙЕСОВСКИЕ ЗАДАЧИ ПОСЛЕДОВАТЕЛЬНОГО РАЗЛИЧЕНИЯ ГИПОТЕЗ ДЛЯ НЕПРЕРЫВНЫХ СЛУЧАЙНЫХ ПРОЦЕССОВ
}

\author{
П. В. ГАПЕЕВ
}

В монографии [1] рассматривается задача последовательного различения двух простых гипотез о среднем значении для винеровского процесса. В данной работе мы рассматриваем указанную задачу для процессов диффузионного типа и фрактального броуновского движения.

Пусть на некотором полном стохастическом базисе $\left(\Omega, \mathscr{F},\left(\mathscr{F}_{t}\right)_{t \geqslant 0}, \mathrm{P}_{\pi}\right)$ задана $\mathscr{F}_{0}$-измеримая случайная величина $\theta$, принимающая два значения, $\mathrm{P}_{\pi}[\theta=1]=\pi, \mathrm{P}_{\pi}[\theta=0]=1-\pi, \pi \in[0,1]$; и существуют меры $\mathrm{P}_{0}$ и $\mathrm{P}_{1}$, локально эквивалентные на фильтрации $(\mathscr{F} t)_{t \geqslant 0}$, сингулярные на $\mathscr{F}_{\infty}=\bigvee_{t \geqslant 0} \mathscr{F}_{t}$, такие, что исходная мера $\mathrm{P}_{\pi}$ имеет структуру $\mathrm{P}_{\pi}=\pi \mathrm{P}_{1}+(1-\pi) \mathrm{P}_{0}$.

Предположим, что наблюдается непрерывно поступающий процесс $X=\left(X_{t}\right)_{t \geqslant 0}$, которьй в зависимости от значения $\theta$ имеет распределения $\mathrm{P}_{i}[X \in A]=\mathrm{P}_{\pi}[X \in A \mid \theta=i], i=0,1$; $A \in \mathscr{F}^{X}=\sigma\left\{X_{t}, t \geqslant 0\right\}$. Будем предполагать, что относительно меры $\mathrm{P}_{\pi}$ случайная величина $\theta$ является взаимно независимой с процессами, имеющими распределения $\mathrm{P}_{i}[X \in A], i=0,1$; $A \in \mathscr{F}^{X}$. Тогда для распределения процесса $X$ относительно меры $\mathrm{P}_{\pi}$ имеет место разложение $\mathrm{P}_{\pi}[X \in A]=\pi \mathrm{P}_{\pi}[X \in A \mid \theta=1]+(1-\pi) \mathrm{P}_{\pi}[X \in A \mid \theta=0], A \in \mathscr{F}^{X}$, где $\pi$ и $(1-\pi)$ являются априорными вероятностями простых статистических гипотез $H_{0}: \theta=0$ и $H_{1}: \theta=1$.

Наша задача заключается в том, чтобъ на основе наблюдений над прочессом $X=$ $\left(X_{t}\right)_{t \geqslant 0}$ последовательно определить, какая из гипотез $H_{0}$ или $H_{1}$ выполнена.

Введем понятие решающего правила $\delta=(\tau, d)$, где $\tau=\tau(\omega)$ является моментом остановки относительно фильтрации $\left(\mathscr{F}_{t}^{X}\right)_{t \geqslant 0}, \mathscr{F}_{t}^{X}=\sigma\left\{X_{s}, s \in[0, t]\right\}, \mathscr{F}_{t}^{X} \subseteq \mathscr{F}_{t}, t \geqslant 0$, a $d=d(\omega)$ есть $\mathscr{F}_{\tau}^{X}$-измеримая случайная величина, принимающая два значения 0 и 1 . Выбор момента остановки $\tau=\tau(\omega)$ означает выбор правила прекращения наблюдений над процессом $X$, а функция терминального решения $d=d(\omega)$ (или решающая функщия) показывает, какую из гипотез в момент $\tau$ нам следует принять. Будем следовать следующему правилу: если $d(\omega)=1$, то принимаем гипотезу $H_{1}$, если $d(\omega)=0$, то утверждаем, что верна гипотеза $H_{0}$.

$\mathrm{C}$ каждым решающим правилом $\delta=(\tau, d)$ можно естественным образом связать потери двух типов: потери, связанные со стоимостью наблюдений, и потери, связанные с неправильным выбором заключительного решения. Средние потери первого типа будем характеризовать величиной $K(\tau)$, где функция $K(t), t \geqslant 0$, имеет вид

$$
K(t)=c_{1} \pi \mathrm{E}_{1} \log \left[d\left(\mathrm{P}_{1} \mid \mathscr{F}_{t}^{X}\right) / d\left(\mathrm{P}_{0} \mid \mathscr{F}_{t}^{X}\right)\right]+c_{0}(1-\pi) \mathrm{E}_{0} \log \left[d\left(\mathrm{P}_{0} \mid \mathscr{F}_{t}^{X}\right) / d\left(\mathrm{P}_{1} \mid \mathscr{F}_{t}^{X}\right)\right],
$$

где математические ожидания являются информационными количествами Кульбака-Лейблера, связанными с мерами $\mathrm{P}_{0}$ и $\mathrm{P}_{1}$, случайная величина $\tau$ означает продолжительность наблюдений и $c_{0}$ и $c_{1}$ являются заданными положительными постоянными, выражающими платы за единицу наблюдений в случае выполнения гипотез $H_{0}$ и $H_{1}$ соответственно. В качестве средних потерь второго типа возьмем величину $a \mathrm{P}_{\pi}[d=0, \theta=1]+b \mathrm{P}_{\pi}[d=1, \theta=0]$, где $a, b>0$ - некоторые заранее выбранные константы, означающие платы за неправильньй выбор решения. Тогда общие средние потери от принятия решающего правила $\delta=(\tau, d)$ задаются равенством $\mathbf{L}_{\pi}(\tau, d)=K(\tau)+\mathrm{E}_{\pi}[a I(d=0, \theta=1)+b I(d=1, \theta=0)]$.

Решение байесовской задачи последовательного различения гипотез состочт в нахождении функции байесовского риска $\rho(\pi)=\inf _{(\tau, d)} \mathbf{L}_{\pi}(\tau, d), \pi \in[0,1]$, и оптимального $\pi$-байесовского решающего правила $\delta^{*}=\delta_{\pi}^{*}=\left(\tau^{*}, d^{*}\right)$, при котором этот инфимум достигается. (Подобную вариационную постановку см. в [2], [3] и [4].) Поставленная задача сводится к задаче об оптимальной остановке процесса апостериорной вероятности $\pi=\left(\pi_{t}\right)_{t \geqslant 0}, \pi_{t}=\mathrm{P}_{\pi}\left[\theta=1 \mid \mathscr{F}_{t}^{X}\right], \pi_{0}=\pi$.

Лемма 1. Функиия байесовского риска $\rho(\pi), \pi \in[0,1]$ имеет представление

$$
\rho(\pi)=\inf _{\tau \in \mathscr{M}} \mathrm{E}_{\pi}\left[k_{\tau}+g_{a, b}\left(\pi_{\tau}\right)\right]=\mathrm{E}_{\pi}\left[k_{\tau}^{*}+g_{a, b}\left(\pi_{\tau^{*}}\right)\right]
$$

əде $g_{a, b}(\pi)=\max \{a \pi, b(1-\pi)\}, \mathscr{M}=\left\{\tau \geqslant 0: \mathrm{E}_{\pi} k_{\tau}<\infty\right\}, k_{t}=c_{0} k_{t}^{0}+c_{1} k_{t}^{1}$, əдe $k^{0}=k^{0}\left(\mathrm{P}_{0}, \mathrm{P}_{1}\right)$ и $k^{1}=k^{1}\left(\mathrm{P}_{1}, \mathrm{P}_{0}\right)$ являются процессами Кульбака мер $\mathrm{P}_{0}$ и $\mathrm{P}_{1}$ и функция 
терминального решения $d^{*}=1$ nри $\pi_{\tau^{*}} \geqslant b /(a+b)$ и $d^{*}=0$ nри $\pi_{\tau^{*}}<b /(a+b)$. (Процесс Кульбака $k(\mathrm{P}, \mathrm{Q})$ мер $\mathrm{P}$ и $\mathrm{Q}$ определяется как возрастающий процесс из разложения Дуба-Мейера относительно меры $\mathrm{P}$ для процесса $\left.\log \left[d\left(\mathrm{P} \mid \mathscr{F}_{t}^{X}\right) / d\left(\mathrm{Q} \mid \mathscr{F}_{t}^{X}\right)\right].\right) 7$

Предположим, что наблюдаемый процесс $X=\left(X_{t}\right)_{t \geqslant 0}$ согласован с фильтрацией $\left(\mathscr{F}_{t}\right)_{t \geqslant 0}$ и относительно исходной меры $\mathrm{P}_{\pi}$ допускает представление

$$
X_{t}=X_{0}+\int_{0}^{t}\left[\theta a_{1}(s, X)+(1-\theta) a_{0}(s, X)\right] d s+\int_{0}^{t} b(s, X) d B_{s},
$$

где $B=\left(B_{t}\right)_{t \geqslant 0}$ - стандартное броуновское движение, функционалы $a_{i}(t, x), i=0,1$ и $b(t, x)$, $t \geqslant 0, x \in C([0, \infty), \mathbb{R})$ являются неупреждающими и выполняется $\mathrm{P}_{\pi}\left[\int_{0}^{t}\left|a_{i}(s, X)\right| d s<\infty\right]=1$, $i=0,1 ; \mathrm{P}_{\pi}\left[\int_{0}^{t} b^{2}(s, X) d s<\infty\right]=1, \mathrm{P}_{\pi}\left[\left(\left[a_{1}(t, X)-a_{0}(t, X)\right] / b(t, X)\right)^{2}>0\right]=1, t \geqslant 0$.

ЛЕмма 2. Предположим, что неупреждающие функциональ $a_{i}(t, x), i=0,1, u b(t, x)$, $x \in C([0, \infty), \mathbb{R})$ удовлетворяют условию Липичца $([2 ;(4.110)])$ и условию линейного роста $([2 ;(4.111)])$. Тогда прочесс апостериорной вероятности $\pi$ имеет дифференциал $d \pi_{t}=\pi_{t}\left(1-\pi_{t}\right)\left[\left(a_{1}(t, X)-a_{0}(t, X)\right) / b(t, X)\right] d \widehat{B}_{t}$, әде прочесс $\widehat{B}=\left(\widehat{B}_{t}, \mathscr{F}_{t}^{X}\right)_{t \geqslant 0}$ является обновляющи броуновским движением: $\mathscr{F}_{t}^{\widehat{B}} \equiv \mathscr{F}_{t}^{X}, t \geqslant 0$, и существует неупреждающ,ий прочесс $\widehat{q}=(\widehat{q}(t, \widehat{B}))_{t \geqslant 0}, \widehat{q}(t, \widehat{B})=\left[\left(a_{1}(t, X)-a_{0}(t, X)\right) / b(t, X)\right]$. Для процесса $k=\left(k_{t}\right)_{t \geqslant 0}$ имеет место выражение $k_{t}=c \int_{0}^{t} \widehat{q}^{2}(s, \widehat{B}) d s, c=\left(c_{0}+c_{1}\right) / 2$.

Рассмотрим процесс $M=\left(M_{t}\right)_{t \geqslant 0}, M_{t}=\int_{0}^{t} \widehat{q}(s, \widehat{B}) d \widehat{B}_{s}$ и замену времени $u(t, \widehat{B})=\langle M\rangle_{t}$. В условиях данной задачи процесс $M$ является непрерывным локальным мартингалом и указанная замена времени является непрерывной, строго возрастающей и имеет обратную замену $v(s, \widehat{B})=\inf \{t \geqslant 0: u(t, \widehat{B})>s\}$ такую, что $v(u(t, \widehat{B}), \widehat{B})=t$ и, следовательно, сушествует неупреждающий функционал $\check{q}(s, x)=\widehat{q}(v(s, x), x), s \geqslant 0, x \in C([0, \infty), \mathbb{R})$.

ЛЕмма 3. Предположим, что для функционала $\breve{q}^{-1}(s, x)=1 / \check{q}(s, x)$ выполняются условие Липшица $([2 ;(4.110)])$ и условие линейного роста $([2 ;(4.111)])$. Тогда прочесс $M$ является обновляющим локальным мартингалом: $\mathscr{F}_{t}^{M} \equiv \mathscr{F}_{t}^{\widehat{B}} \equiv \mathscr{F}_{t}^{X}, t \geqslant 0$.

С помощью замены времени $u(t, \widetilde{B})=\langle M\rangle_{t}$ в процессе $\pi=\left(\pi_{t}\right)_{t \geqslant 0}$ данная задача сводится к задаче последовательного различения гипотез для винеровского процесса (cм. $[1 ; \mathrm{IV}$, п. 2]).

ТеОрема 4. Предположим, что выполнены все условия предыдущих лемм. Тогда функция байесовского риска $\rho(\pi), \pi \in[0,1]$, имеет единственное представление

$$
\rho(\pi)=a A_{*}+\left(\pi-A_{*}\right)\left[a-c \psi\left(A_{*}\right)\right]+c\left[\Psi(\pi)-\Psi\left(A_{*}\right)\right], \quad c=\left(c_{0}+c_{1}\right) / 2,
$$

$\Psi(\pi)=(1-2 \pi) \log \frac{\pi}{1-\pi}, \psi(\pi)=\Psi^{\prime}(\pi)=\left(\frac{1-\pi}{\pi}-\frac{\pi}{1-\pi}\right)+2 \log \frac{1-\pi}{\pi}, \pi$-байесовское решающее правило $\delta^{*}=\left(\tau^{*}, d^{*}\right)$ существует и имеет вид: $\tau^{*}=\inf \left\{t \geqslant 0: \pi_{t} \notin\left(A_{*}, B_{*}\right)\right\} ; d^{*}=1$ при $\pi_{\tau^{*}}=B_{*}, d^{*}=0$ при $\pi_{\tau^{*}}=A_{*} ;$ и границы $A_{*}$ и $B_{*}$ однозначно определяются из следующей системы: $\left\{\begin{array}{l}b+a=c\left[\psi\left(A_{*}\right)-\psi\left(B_{*}\right)\right], \\ b\left(1-B_{*}\right)=a A_{*}+\left(B_{*}-A_{*}\right)\left[a-c \psi\left(A_{*}\right)\right]+c\left[\Psi\left(B_{*}\right)-\Psi\left(A_{*}\right)\right] .\end{array}\right.$

ЗАмечАниЕ 5 . В случае, если наблюдаемый процесс $X=\left(X_{t}\right)_{t \geqslant 0}$ имеет представление $X_{t}=\theta t+B_{t}^{H}$, где $B^{H}=\left(B_{t}^{H}\right)_{t \geqslant 0}$ - фрактальное броуновское движение с показателем автомодельности Харста $H \in(0,1)$, утверждение теоремы 4 остается справедливым.

\section{СПИСОК ЛИТЕРАТУРЫ}

[1] Ширяев А. Н. Статистический последовательный анализ. М.: Наука, 1976. [2] Липцер Р.Ш., Ширяев А. Н. Статистка случайных процессов. М.: Наука, 1974. [3] Koell C. // Sequential Anal. 1994. V. 13. № 3. P. 221-236. [4] Galtchouk L. I. Optimality of the Wald SPRT for processes with continuous time parameter // Submitted in Annals of Statistics. 